\title{
EL PAPEL DEL HISTORIADOR DEL ARTE EN LA INTERVENCIÓN EN EL PATRIMONIO CULTURAL ${ }^{1}$
}

\author{
GONZALO M. BORRÁS GUALIS
}

Profesor Emérito de Historia del Arte de la Universidad de Zaragoza

\section{Preludio}

Debo señalar en primer lugar que la preocupación de los historiadores del arte por la problemática del patrimonio cultural no es nueva, aunque hoy siga en pleno vigor. Las referencias podrían remontarse a fechas lejanas, pero baste anotar aquí, a nivel internacional, la sección dedicada a "El papel de la Historia del Arte en las conservación del Patrimonio" en el XXVI Congreso del Comité Internacional de Historia del Arte (CIHA), celebrado en Washington en 1985, y a nivel nacional las memorables Jornadas que el Comité Español de Historia del Arte (CEHA), con la colaboración del Instituto Andaluz del Patrimonio Histórico, dependiente de la Consejería de Cultura de la Junta de Andalucía, organizó en la ciudad de Cádiz los días 17 al 19 de junio de 1992, bajo el título de Historia del Arte y Bienes Culturales $^{2}$ cuyo objetivo monográfico fue abrir "un debate en torno a la presencia de los historiadores del arte en los proyectos de conservación del Patrimonio".

${ }^{1}$ Texto de la conferencia pronunciada en el master en Historia del Arte de la Universidad de Córdoba el 20 de octubre

2 A estas jornadas de Cádiz fui invitado por el profesor Alfredo Morales, de la Universidad de Sevilla, y participé con una ponencia sobre "Fundamentos para la profesionalización de la Historia del Arte en España". Las Actas tardaron seis años en publicarse con el título CUADERNOS. Historia del Arte y Bienes Culturales, Instituto Andaluz del Patrimonio 
Los simposios, jornadas o congresos promovidos específicamente por los historiadores del arte sobre la problemática actual del Patrimonio han menudeado por doquier en los últimos años en las diferentes comunidades autónomas; así, por ejemplo, en tierras aragonesas se celebraba en Alcorisa (Teruel) durante los días 23 al 25 de septiembre de1993 el VIII Coloquio de Arte Aragonés ${ }^{3}$, promovido por el Departamento de Historia del Arte de la Universidad de Zaragoza, con la colaboración del Gobierno de Aragón, y dedicado a la problemática de las intervenciones en el Patrimonio histórico-artístico de Aragón, cuyas actas no se editaron.

Otro congreso, éste de alcance nacional como el de Cádiz, y siguiendo su modelo de colaboración entre el CEHA y, en este caso, la dirección General del Patrimonio Histórico del Gobierno de Canarias, se celebró con éxito en la Universidad de La Laguna en Tenerife, durante los días 23 al 26 de mayo de 1994, con el título Hacia una conciencia sobre el Patrimonio ${ }^{4}$, dirigido por el profesor Alberto Darias Príncipe, una vez más sin edición de actas, aunque se alcanzase a firmar por todos los participantes una importante declaración institucional en los siguiente términos:

"Es necesario que los historiadores del arte, en el actual momento de aplicación y desarrollo de los nuevos planes de estudio de la Licenciatura en Historia del Arte en las universidades españolas, sepamos dar una respuesta adecuada a la problemática social de la conservación del patrimonio cultural, de modo que tanto la docencia como la investigación universitarias en Historia del Arte se doten de una metodología operativa, que tenga como horizonte y fundamento ético de nuestra disciplina la conservación del patrimonio".

Y este creciente interés de los historiadores del arte ante la problemática actual del Patrimonio se mantuvo vivo durante los años siguientes. Así durante los días 10 y 11 de octubre de 1997, el CEHA dedicaba un Simposio a la figura del historiador y crítico de arte Juan Antonio Gaya Nuño, celebrado en la ciudad de Soria con la colaboración de Caja Duero y organizado por el que suscribe y la profesora Lourdes Cerrillo Rubio. Una de las ponencias de este Simposio, cuyas actas a diferencia de los casos anteriores han sido editadas, estuvo desarrollada por el profesor Alfredo Morales Martínez, catedrático de Historia del Arte de la Universidad de Sevilla, de nuevo con el tema "El historiador del arte ante la actual problemática del patrimonio cultural"s.

Tras el Simposio de Soria, y sin cesura, durante los días 16 al 18 de octubre de 1997, la Dra. María del Mar Lozano Bartolozzi, Catedrática de Historia del Arte de la Universidad de Extremadura, coordinaba un seminario interdisciplinar sobre Turismo, Conservación y Rebabilitación del Patrimonio Arquitectónico y Artístico, organizado conjuntamente por la Fundación La Caixa y la Universidad de Extremadura en torno a la exposición Vivir las Ciudades Históricas y desarrollado en la ciudad de Cáceres ${ }^{6}$. La coordinadora tuvo la amabilidad

Histórico, Junta de Andalucía, Consejería de Cultura, 1998, pp. 50-55. Algunas de las reflexiones allí planteadas las he ido desgranando en varios artículos de la revista ARTIGRAMA, del Departamento de Historia del Arte de la Universidad de Zaragoza; véase " A modo de Presentación: El papel del historiador del arte en la conservación y restauración de monumentos y obras artísticas", ARTIGRAMA, 6-7 , 1989-1990, pp. 7-12; "A modo de Presentación: El Museo, una asignatura pendiente para los historiadores del arte", ARTIGRAMA , 8-9, 1991-1992, pp. 7-11; y "La Historia del Arte en la encrucijada”, ARTIGRAMA, 10, 1993, pp. 45-53. Por todo ello resultan inevitables bastantes reiteraciones al volver sobre el mismo tema con carácter general, por lo que ruego disculpas a quienes ya estén familiarizados con mi pensamiento sobre la materia

${ }^{3}$ En este VIII Coloquio de Arte Aragonés fui invitado a participar con una ponencia sobre "El papel de Historiador del Arte en la intervención en el Patrimonio Histórico-Artístico".

${ }^{4}$ A este Congreso de la Universidad de La Laguna fui invitado por el profesor Alberto Darias y participé con una ponencia sobre "La investigación operativa en Historia del Arte en relación con el Patrimonio".

${ }^{5}$ Véase Actas. Simposio El historiador del Arte, hoy. 10 y 11 de octubre de 1997. Soria. CEHA, Caja Duero, [1998], pp. 119-126. Estas Actas fueron presentadas durante el Simposio del CEHA sobre "José Camón Aznar y la historiografía artística de su tiempo" celebrado en Zaragoza los días 17 y 18 de abril de 1998

${ }^{6}$ Véase Vivir las ciudades históricas. Seminario. Turismo, conservación y rehabilitación del patrimonio arquitectónico y artístico. Cáceres, 16, 17 y18 de octubre de 1997. Institución Cultural El Brocense, Cáceres, 1998, pp. 39-45 
de invitarme a participar con una ponencia en la que una vez más se tratase el particular punto de vista del historiador del arte en relación con la problemática actual del Patrimonio y al mismo tiempo se apuntasen algunas sugerencias y propuestas sobre "El papel del Historiador del Arte en la intervención en el Patrimonio", título que coincide con el de esta conferencia.

Se trata, pues de un tema de acuciante actualidad en la década de los noventa del pasado siglo y bastante recurrente, no sólo en los reuniones científicas promovidas por los historiadores del arte, sino en todo tipo de simposios de carácter interdisciplinar en los que cada vez ha sido más notable por fortuna la presencia, participación y defensa de los puntos de vista de los historiadores del arte.

Transcurrida ya más de una década, conviene insistir en que en la gestión del patrimonio cultural el historiador del arte concurre con numerosos profesionales de intereses muy variados, por lo que resulta oportuno iniciar nuestra reflexión afirmando que el historiador del arte no pretende una vana e inútil competencia con ninguna otra disciplina, queriendo atenerse estrictamente a la función del historiador del arte, con planteamientos específicos sobre el tema, que son aquellos que hay que considerar prioritariamente y aquellos que únicamente legitiman nuestro concurso.

\section{La Historia del Arte y los Bienes Culturales.}

Entendemos hoy la Historia del Arte ${ }^{7}$ como una historia de las obras de arte valoradas como hechos históricos, de acuerdo con unos criterios de autenticidad y de calidad, que comportan un doble juicio de valor, tanto histórico como crítico. Hay que ser historiadores y críticos en el mismo juicio de valor; no cabe Historia del arte sin Crítica, ni tampoco Crítica de arte sin Historia. Nuestra tarea como historiadores del arte radica en establecer estos juicios de valor sobre las obras de arte, interpretar las obras de arte como hechos históricos, es decir, explicar en qué medida han contribuído a configurar una cultura.

Pero la Historia del Arte como historia de las obras de arte no ha hecho a lo largo de los dos últimos siglos sino ampliar sin interrupción el objeto de su estudio, de modo que a las tres manifestaciones artísticas tradicionales de la arquitectura, la escultura y la pintura se han ido sumando otras muchas. Así a lo largo del siglo XIX -a partir de movimientos como Arts and Crafts y de estudios como los de Alois Riegl- se recuperan críticamente para la Historia del Arte en la cultura occidental el numeroso grupo de las artes decorativas, también llamadas por algunos artes aplicadas e industriales. Desde entonces las piezas de plata, las joyas, los bronces, los tapices y alfombras, los bordados, los marfiles, la cerámica decorada, los vidrios, los esmaltes, los muebles y las armaduras de madera, y una larga relación que alcanza hasta los objetos de diseño industrial, todas estas manifestaciones mencionadas, tanto en versión "popular" como en versión "culta", pueden alcanzar la valoración de obra de arte y convertirse en objeto de estudio para los historiadores del arte.

En este proceso abierto la Historia del Arte ha seguido ampliando su objeto de estudio a lo largo del siglo XX incorporando al mismo los medios icónicos de masas, como la fotografía, el cartel, el cómic o historieta, el cine, la televisión y el video, que a diferencia de los lenguajes artísticos tradicionales han de ser considerados como las auténticas obras de arte del siglo XX.

Y queda por último, aunque no en último lugar, la recuperación crítica de la ciudad por la Historia del Arte, la ciudad entendida como un continuo espacio temporal, como un sistema abierto configurado por factores espaciales e históricos. Desde los estudios de Pierre Lavedan, entre los franceses, hasta los del profesor Antonio Bonet Correa o de María del Mar Lozano Bartolozzi, entre los españoles, los historiadores del arte hemos ido aportando en relación con la interpretación y valoración de la ciudad unos enfoques muy diferentes a los puntos de vista de los ingenieros y arquitectos, de los geógrafos, de los juristas, de los

\footnotetext{
${ }^{7}$ Un desarrollo más extenso sobre la evolución del concepto de Historia del Arte en la modernidad y sobre la constante ampliación de su objeto de estudio puede seguirse en mi manual sobre Teoría del Arte I, vol. 11 de la col. "Conocer el Arte", Madrid, Historia 16, 1996
} 
sociólogos, de los filósofos, y de toda la amplia gama de profesionales que concurren en la ciencia del urbanismo y de la planificación urbana. Incluso ha llegado a formularse un planteamiento integral de la Historia del Arte como historia de la ciudad por parte del profesor italiano Giulio Carlo Argan.

En suma, la verdadera tarea del Historiador del Arte es la construcción de la Historia del Arte, o sea, dedicarse al estudio y valoración de las obras de arte como hechos históricos, instalándose en un nivel especulativo, de investigación pura.

Pero a la condición de las obras de arte la cultura de nuestro tiempo ha añadido un valor nuevo, el valor de patrimonio artístico ${ }^{8}$, que no está comprendido en el concepto inicial de obra de arte, estimando de este modo las obras de arte como bienes de carácter patrimonial, un patrimonio artístico que pertenece a toda la humanidad y que ha de ser conservado y transmitido a las generaciones futuras. Diversas Instituciones Culturales, tanto de ámbito internacional como nacional, han desarrollado en los últimos cincuenta años el concepto de patrimonio artístico, que se ha ido expandiendo en ondas concéntricas cada vez de mayor radio, ampliando sucesivamente el ámbito de bienes protegidos a todos los bienes de la cultura, tanto material como espiritual. Así se ha ido pasando sin solución de continuidad del concepto de patrimonio artístico al más amplio de patrimonio histórico-artístico y al actual de patrimonio cultural o Bienes de Interés Cultural (BIC).

En consecuencia el Historiador del Arte se ha encontrado con que las obras de arte, objeto del estudio de la Historia del Arte, donde han de ser valoradas como hechos históricos, han adquirido junto con otras muchas manifestaciones de la cultura esta nueva dimensión de bienes culturales, un aspecto añadido y sobreimpuesto que no sólo no puede ser ignorado sino al que la sociedad está concediendo una atención prioritaria.

Hasta tal punto se ha ido imponiendo socialmente el interés por los Bienes Culturales que la Historia del Arte ha debido superar una difícil encrucijada en relación con la problemática global del Patrimonio Cultural, hasta el punto de que los estudios universitarios de Historia del Arte han podido correr un serio peligro de desnaturalización. En este sentido, por un lado, los nuevos planes de estudio de la Licenciatura en Historia del Arte están concedido amplio acomodo a todo un conjunto de materias orientadas a dar una respuesta a esta creciente demanda social: Museología, Conservación y Restauración de obras de arte, Legislación sobre Patrimonio Cultural y un amplio etcétera. Por otro lado ha intentado una nueva licenciatura en Conservación y Restauración de Bienes Culturales, al tiempo que aumentan las ofertas de postgrado en esta misma materia.

Ante todo quiero reconocer que es explicable que la Historia del Arte en España no quiera perder de nuevo la oportunidad - tal vez la última- de lograr una salida profesional específica, teniendo en cuenta que ya se han malogrado demasiadas oportunidades en el inmediato pasado. En efecto ha transcurrido ya más de un cuarto de siglo desde la incorporación de nuestra titulación específica de Licenciado en Historia del Arte en la universidad española, sin que a la misma haya correspondido la exigencia legal de dicha titulación para ejercer la docencia de la materia en la enseñanza media, vacío legal que ha constituido nuestro principal talón de Aquiles en las salidas profesionales de los historiadores del arte.

En efecto, los licenciados en Geografía y en Historia nos han aventajado a la hora de preparar los temarios de oposiciones a plazas de profesores de enseñanza media para la docencia conjunta de la Geografía, de la Historia y de la Historia del Arte, al existir una sola titulación para las tres materias, en la que los temas de Geografía e Historia se llevan la parte del león. Tampoco parece que la situación vaya a mejorar mucho con la opción artística del nuevo Bachillerato, para cuyas plazas docentes el perfil de la licenciatura en Bellas Artes se configura asimismo muy competitivo.

Otra salida profesional malograda ha sido la del Cuerpo Técnico Facultativo de Museos; debido a la vinculación legal de estos funcionarios a las excavaciones arqueológicas

\footnotetext{
${ }^{8}$ Para un tratamiento más profundo de la evolución del concepto de Patrimonio Artístico puede consultarse el manual del profesor Alfredo J. MORALES sobre Patrimonio Histórico-Artístico, vol. 13 de la col. "Conocer el Arte", Madrid, Historia 16, 1996
} 
provinciales, nos ganaron esta vez la mano los arqueólogos, situación que se ha arrastrado durante demasiado tiempo con evidente injusticia para los fondos artísticos de nuestros Museos, y de la que en la actualidad se está saliendo paulatinamente. Otra posible salida profesional, la del expertizaje artístico reglado, no fue más allá de los buenos propósitos de un Director General de Bellas Artes. Malogradas ya tantas opciones de salida profesional en el inmediato pasado, parecen lógicos nuestra preocupación e interés por la actual demanda en Conservación y Restauración de Bienes Culturales e incluso nuestro estado de necesidad podría propiciar un abrazo precipitado del "bienismo" cultural.

Antes de exponer mis reticencias en relación con el "bienismo" cultural y para que no se malinterpreten mis puntos de vista en esta cuestión, deseo proclamar que siempre he estimado irrenunciable el compromiso de la Universidad con la sociedad de su tiempo. Es más, pienso, en concreto, que una Historia del Arte que no sea capaz de dar respuesta adecuada a las exigencias sociales de cada momento, ha de considerarse caduca. La demanda editorial de nuestra disciplina - en su mayor parte de obras de síntesis y divulgación-, ha constituido una buena piedra de toque en las últimas décadas; muchos de nosotros hemos dado una decidida respuesta de estrecha colaboración con el mercado editorial sin preocuparnos en exceso del descrédito académico que para muchos conlleva este tipo de trabajo.

Pero un compromiso ético con la sociedad no debe desnaturalizar el papel específico de cada disciplina universitaria, antes al contrario, ésta debe proyectar un foco de luz propia sobre la realidad circundante. En definitiva, lo que la sociedad necesita y puede exigir a un historiador del arte es que éste se comporte como tal. En este contexto de pensamiento se inscribe mi posición sobre el papel que le corresponde al historiador del arte en relación con la actual demanda social de Conservación y Restauración de Bienes Culturales, para la que se acaba de proponer una nueva licenciatura universitaria, que daría acceso al título de "bienista".

He mantenido una opinión beligerantemente adversa al proyecto de una licenciatura universitaria en bienismo cultural. Estimo que, en función de cada Bien de Interés Cultural a preservar, deben exigirse con carácter previo licenciaturas en Historia, Historia del Arte, Arquitectura, Bellas Artes, etcétera, si queremos evitar una profesión vacía de contenido, puramente mecánica, desconocedora de todo lo relativo al objeto que perpetra manipular. ¿Ha pensado alguien en qué devendrían unas titulaciones médicas -en cirugía, ginecología, pediatría, etcétera- para las que previamente no se exigiese una licenciatura en Medicina? ¿Nadie ha reparado en la vacuidad de unos técnicos de la información carentes de cualquier conocimiento sobre el que informar al público? La formación de los "bienistas", si no se quiere desvirtuar, ha de plantearse con carácter de especialidad para postgraduados universitarios, formación que bien pudiera quedar encomendada al Instituto del Patrimonio Histórico español, antes denominado Instituto de Conservación y Restauración de Bienes Culturales.

\section{El papel específico del historiador del arte en los diferentes niveles de intervención en el Patrimonio'.}

De todo lo afirmado en el epígrafe anterior no debe deducirse en absoluto que el historiador del arte haya de quedar al margen de la intervención en el Patrimonio Cultural en los tres niveles "operativos" del examen, la preservación y la restauración. Nada más alejado de nuestro propósito.

Precisamente para la actividad específica del historiador del arte pueden establecerse asimismo otros tres niveles, de carácter especulativo, cuya concurrencia es necesaria y previa a cada uno de los tres niveles operativos de la in tervención en el Patrimonio Cultural. Estos niveles son la investigación pura, la catalogación y la interpretación de los valores histórico-

\footnotetext{
${ }^{9}$ Esbocé por vez primera el contenido de este epígrafe en el VIII Coloquio de Arte Aragonés, celebrado en Alcorisa, los días 23 a 25 de septiembre de 1993, cuyas actas, como se ha dicho, no fueron editadas
} 
artísticos, que deben preceder siempre y acompañar con carácter obligatorio al examen, a la preservación y a la restauración.

Quiero significar que jamás se debería proceder a la realización de un "examen" de un Bien Cultural sin el acompañamiento preceptivo del historiador del arte que haya realizado la investigación pura previa de dicha obra de arte. ; Cuántos aspectos dejan de apreciarse en un examen previo, aparentemente tan inocuo desde en punto de vista de la intervención - y luego con tantas consecuencias-, tan sólo por no contar con la mirada del historiador del arte, una mirada fundamentada en la investigación previa, que le permite apreciar lo que otros ojos no ven en una exploración preliminar! i Cuántas veces se recurre tarde y mal al historiador del arte! ¿Cuando se contará habitualmente con el historiador del arte desde el primer nivel de la intervención en el Patrimonio Cultural, desde las primeras exploraciones y exámenes? Nunca insistiremos demasiado en la necesidad de esta presencia previa del historiador del arte y en las consecuencias de la misma para los siguientes niveles de la intervención en el Patrimonio Cultural.

Por lo que se refiere a la catalogación artística como primer paso en la tutela del Patrimonio Cultural y en todo caso como actividad específica del historiador del arte, previa y necesaria para cualquier proyecto de preservación de un Bien Cultural, parece que se trata de la tarea en la que hemos logrado un mayor grado de reconocimiento profesional. Pero no es posible tan siquiera esbozar en este momento la compleja historia de la redacción de los Catálogos Monumentales de España, de los Inventarios Artísticos y de los actuales Inventarios de Bienes Muebles de la Iglesia Católica Española, ninguno de ellos concluidos en su totalidad ${ }^{10}$. Baste anotar que, atendida la enorme complejidad del estado actual de la catalogación artística, en este nivel la concurrencia del historiador del arte ha devenido imprescindible y es reconocida interdisciplinarmente.

Sin duda el mayor énfasis ha de ser puesto en nuestro tercer nivel especulativo, en el de la interpretación de los valores histórico-artísticos, como un informe necesario que debe acompañar con carácter previo a cualquier proyecto o memoria de restauración. Debemos defender que la presencia técnica del historiador del arte mediante este informe previo sea exigible por ley, o al menos por exigencia administrativa, y no responda tan sólo a la hospitalidad o cortesía interdisciplinar de los actuales técnicos responsables de la memoria de restauración.

Pero a la vez hemos de lograr que este informe histórico-artístico no constituya por más tiempo una inane relación de acarreo de datos, carentes de valoración y de interpretación. En este nivel es básico que el historiador del arte no pierda nunca de vista cual es su auténtico papel, una función que ningún otro profesional va a realizar en su lugar: la de poner de relieve los valores artísticos y por tanto históricos del Bien Cultural a preservar, su interpretación cultural, emitiendo juicios de valor sobre el mismo en los que se puedan fundamentar los criterios a adoptar en los diferentes proyectos de intervención cultural.

Si el historiador del arte se atiene a dichas actividades específicas de investigación, catalogación e interpretación, previas y necesarias para los tres niveles operativos de intervención en el patrimonio, no sólo nuestra colaboración será respetada y requerida por los demás profesionales, sino que difícilmente nuestro quehacer podrá entrar en colisión con ninguno.

\section{Apostillas sobre el Turismo Cultural y sobre la gestión del Patrimonio Cultural.}

Para concluir ruego me sean aceptadas un par de apostillas sobre el Turismo Cultural y sobre la gestión del Patrimonio Cultural.

${ }^{10}$ Constituye una excepción y un paradigma el Catálogo monumental de Navarra, dirigido por la profesora Concepción GARCÍA GAINZA, que ha sido realizado y editado en su totalidad. Véase "El Catálogo monumental de Navarra, dirigi-do por Concepción García Gainza”, en Gonzalo M. BORRAS GUALIS, Cómo y qué investigar en historia del arte. Una crítica parcial de la historiografía del arte española, Ediciones del Serbal, Barcelona, 2001, pp. 74-75. 
La primera referencia parece que haya de encontrar un acomodo natural en este simposio interdisciplinar, que en el enunciado de sus contenidos antepone el término Turismo a los de Conservación y Rehabilitación del Patrimonio, por lo que se infiere que en este foro existe un interés prioritario en la reflexión sobre la problemática del Turismo Cultural en la actualidad, y por lo tanto se nos demanda aquella visión específica que los historiadores del arte podamos aportar a este tema.

Es evidente que el Patrimonio Cultural en su conjunto - desde la naturaleza hasta el artese está erigiendo paso a paso en una de las ofertas turísticas más atractivas del momento. Aunque esta demanda de un turismo cultural de calidad no es nueva, habiéndose desarrollado tímidamente en España desde hace algún tiempo como réplica al desastroso episodio del masivo turismo de sol en las costas, circunstancia que incitó a muchos consumidores a volver la mirada hacia otras alternativas de ocio menos enajenantes. En este línea siempre me parecieron dignas de encomio las intervenciones realizadas por César Manrique -y continuadas hoy por la Fundación que lleva su nombre- en la isla de Lanzarote para inducir un turismo cultural de calidad.

En el momento actual se intensifican de nuevo en algunas comunidades autónomas las apuestas por el Patrimonio Cultural como motor del desarrollo turístico. Aragón, la comunidad autónoma a la que pertenezco, no anda a la zaga en cuanto a ideas originales sobre desarrollo del Turismo Cultural. Ahí está ya la magnífica realidad de los llamados Parques Culturales -los del Maestrazgo, de Albarracín, del río Martín ${ }^{11}$ y del río Vero-, con una consideración integrada de todo el patrimonio cultural de una comarca como oferta turística: desde la naturaleza al arte, desde la arqueología al folklore, todos los recursos culturales de un territorio son ofrecidos como un paquete global de extraordinario atractivo.

Asimismo es oportuno recordar que el turismo cultural puede ser objeto de perversión con estrategias de explotación masiva. A la memoria de todos acuden algunos casos extremos, como las visitas turísticas a La Alhambra y al Generalife de Granada, o a la última edición de las exposiciones sobre las Edades del Hombre en la diócesis del Burgo de Osma, en los que la avalancha autorizada de visitantes ha puesto fin al disfrute y a la contemplación sosegadas.

En este sentido debemos recordar que tanto la UNESCO como el Consejo de Europa han elaborado un "corpus" doctrinal sobre las políticas del Patrimonio Cultural de obligado cumplimiento para España. La Declaración de Ministros de Helsinki (1996) ha propuesto la "aplicación de estrategias sostenibles para el turismo cultural". Es obvio que la pujanza del turismo puede contribuir al desarrollo y a la revitalización de las regiones, pero, a la vez, como hemos ya denunciado, es susceptible de crear presiones sobre el patrimonio cultural que afecten a la calidad de vida de sus comunidades. Las políticas y estrategias de turismo cultural deben estar encuadradas en la perspectiva de una "utilización equilibrada y sostenible del patrimonio" reservando las posibilidades de uso de este patrimonio para las generaciones venideras.

Me resta una última apostilla dedicada a la gestión del Patrimonio Cultural, actividad que en principio pudiera parecer bastante alejada del papel del historiador del arte, sobre el que aquí estamos reflexionando. Personalmente entiendo que en el vasto, complejo e interdisciplinar campo de profesiones relacionadas con el Patrimonio ya existen por fortuna tanto conservadores como restauradores de acreditada solvencia, así como una docencia enfocada específicamente a la formación de conservadores y restauradores. Pero la existencia de estas enseñanzas y de estos titulados en Conservación y en Restauración no resuelve en absoluto el principal problema del Patrimonio Cultural en nuestros días, que es un problema de gestión.

11 Véase Antonio BELTRAN MARTINEZ et al.: Guía del Parque Cultural del Río Martín. Zaragoza, Prames, 1997. En la Introducción se explica este nuevo concepto de parque cultural. Una visión reciente del tema puede seguirse en Pedro Luis HERNANDO SEBASTIÁN, "Los parques culturales de Aragón", HERITAGE \& MUSEOLOGY, 8, septiembre-octubre 2011, pp. 88-95. 
A mi entender la carencia esencial del Patrimonio Cultural en el momento actual es la de profesionales de la gestión. Hacen falta unos profesionales que con un amplio conocimiento de la problemática del Patrimonio Cultural en todos sus aspectos (legislación, documentación y catálogo, conservación y restauración, y difusión cultural) no van a dedicarse profesionalmente a ninguno específico, sino a la gestión, es decir, a la elaboración y ejecución de planes de actuación sobre el Patrimonio Cultural, para lo cual es necesario además de lo dicho un profundo conocimiento de la gestión en todas sus facetas: política, administrativa, económica, etc.

Aquí, en la gestión, existe un vastísimo campo de trabajo para bastantes profesionales relacionados con el Patrimonio; también, por supuesto, para aquellos historiadores del arte que quieran optar por esta dedicación. Tan sólo una buena gestión del Patrimonio Cultural ${ }^{12}$ podrá arbitrar soluciones eficaces a los muchos problemas que la conservación del Patrimonio Cultural plantea en nuestros días.

\section{Conclusión}

De todo lo dicho se desprende el importante reto que la problemática del Patrimonio Cultural, como valor añadido a la obra de arte, plantea en la actualidad a los historiadores del arte. Creo haber marcado con precisión los límites de nuestro papel de historiadores del arte en relación con el Patrimonio: por un lado, una Historia del Arte que no sea capaz de dar respuestas adecuadas a las exigencias sociales de cada momento ha de considerarse caduca; pero, por otro, nuestra específica tarea como historiadores del arte radica en la interpretación de los valores histórico-artísticos de las obras de arte. Entre ambos límites puede extenderse un vasto campo de acción.

${ }^{12}$ La Universidad de Zaragoza aprobó a fines de 1997 un estudio propio de postgrado sobre "Gestión del Patrimonio Cultural", cuya primera edición se desarrolló en el año 1999 entre los meses de febrero a noviembre, bajo la coordinación del que suscribe. Las dos siguientes ediciones se hicieron con carácter trienal en los años 2002 y 2005, respectivamente. En el momento actual, con la titulación académica de master, se desarrolla con carácter anual, aunque mantiene el formato inicial del año 1999 